\title{
Avaliação do Sistema Inseto Praga-Parasito Nativo-Parasito Exótico por Meio da Dinâmica de Populações
}

S. TERNES, Embrapa Informática Agropecuária, Cx.P. 6041, 13083-886 Campinas, SP, Brasil

H.M. YANG, Departamento de Matemática Aplicada, Instituto de Matemática, Estatística e Computação Científica, UNICAMP, Cx.P. 6065, 13081-970 Campinas, SP, Brasil.

\begin{abstract}
Resumo. Neste trabalho desenvolve-se um modelo matemático compartimental e determinístico para analisar a dinâmica populacional da interação do inseto-praga Phyllocnistis citrella com seus inimigos naturais Galeopsomyia fausta e Ageniaspis citricola. Através do estudo analítico do sistema de equações, avalia-se a possibilidade de coexistência entre as espécies e a competição indireta entre os dois inimigos naturais.
\end{abstract}

\section{Introdução}

A larva-minadora-da-folha-dos-citros, Phyllocnistis citrella (Lepidoptera: Gracillariidae), é um inseto-praga de grande importância para a citricultura nacional. Foi encontrada pela primeira vez no Brasil em março de 1996 no Estado de São Paulo, disseminando-se rapidamente para outros Estados do país, como Bahia e Minas Gerais, ainda no mesmo ano [1].

A larva penetra nas folhas da planta levando à atrofia do tecido foliar. Além do comprometimento à fotossíntese, os danos provocados podem resultar na queda prematura das folhas, impedindo o desenvolvimento de brotações e, conseqüentemente, refletindo no potencial produtivo do pomar. Além disso, os ferimentos provocados pela larva à folha e outros órgãos da planta favorecem a penetração da bactéria causadora do cancro cítrico, Xanthomonas axonopodis pv. citri [1].

A espécie Galeopsomyia fausta (Hymenoptera: Eulophidae) foi identificada como o principal parasito nativo da $P$. citrella no Estado de São Paulo, onde chegou a índices de $90 \%$ entre os parasitos nativos coletados em campo [3]. A G. fausta é um ectoparasito da fase de pupa e sua reprodução é do tipo partenogênese telítoca (óvulo não fecundado produz apenas fêmeas), apresentando machos ocasionais. A fêmea da G. fausta ovipõe sobre a câmara pupal da P. citrella. O ovo eclode dando origem à larva que se alimenta da pupa do hospedeiro, ocasionando a sua morte. 
A espécie Ageniaspis citricola (Hymenoptera: Encyrtidae) foi introduzida no Brasil em 1998 para o controle biológico da P. citrella [2], e é um endoparasito de ovos e larvas jovens. O parasitismo é gregário, podendo produzir até oito ovos por hospedeiro. Os ovos não fertilizados produzem machos (partenogênese arrenótoca) e ovos fertilizados produzem machos e fêmeas.

O objetivo deste trabalho é apresentar um modelo matemático compartimental e determinístico para analisar a dinâmica populacional do inseto-praga Phyllocnistis citrella em interação com seus inimigos naturais Galeopsomyia fausta (parasito nativo) e Ageniaspis citricola (parasito exótico). Através do estudo analítico do sistema de equações, avalia-se a possibilidade de coexistência entre as espécies e a competição indireta entre os dois inimigos naturais.

\section{Formulação do modelo}

O modelo matemático proposto visa descrever a dinâmica populacional do insetopraga Phyllocnistis citrella em um processo de parasitismo pelos inimigos naturais Galeopsomyia fausta e Ageniaspis citricola [7].

A abordagem utilizada caracteriza-se pelo desenvolvimento de modelos compartimentais estágio-estruturados [5]. Cada compartimento do modelo representa uma fase do ciclo de vida do inseto. Assim, o processo que representa as entradas e saídas em cada fase, ou estágio do ciclo, é descrito matematicamente por uma equação diferencial ordinária.

No modelo proposto consideram-se todas as fases principais do ciclo de vida diretamente envolvidas no processo de parasitismo. As demais fases são englobadas em parâmetros apropriados. Tal simplificação permite a manipulação algébrica do sistema de equações diferenciais resultante e a conseqüente obtenção de importantes resultados analíticos, sem comprometer a representação do fenômeno biológico.

$\mathrm{Na}$ construção do modelo geral e na definição dos compartimentos considerou-se que: (a) o modelo representa a população de fêmeas ao longo do tempo, podendo ser estimada a população de machos através da razão sexual de cada espécie; (b) os compartimentos dos modelos representam as fases do ciclo de vida de cada espécie envolvidas diretamente no processo de parasitismo. As outras fases são incorporadas em parâmetros apropriados; (c) o inimigo nativo Galeopsomyia fausta é um parasito generalista, de tal modo que a população dessa espécie mantém um crescimento natural independente do nível populacional da praga; (d) os períodos de cópula, pré-oviposição, maturação e viabilidade dos ovos estão considerados conjuntamente na taxa de natalidade. Assim, a fase de ovo não é representada num compartimento em separado nos modelos, contribuindo para a simplificação dos mesmos e (e) a fase de larva não é subdividida em estádios, ou seja, é considerada uma fase larval única que engloba todos os estádios. De modo semelhante, as fases de pré-pupa e pupa são consideradas como única.

Com base nessas hipóteses, as variáveis dinâmicas consideradas no modelo são as fases de larva $\left(\mathrm{L}_{m}\right)$, pupa $(\mathrm{P})$ e adulta $(\mathrm{M})$ para o inseto-praga; as fases de larva $\left(\mathrm{L}_{g}\right)$ e adulta $(\mathrm{G})$ para o parasito nativo e as fases de larva $\left(\mathrm{L}_{a}\right)$, fêmea virgem $\left(\mathrm{A}_{v}\right)$ 
e fêmea acasalada (A) para o parasito exótico. A Figura 1 mostra o esquema de compartimentos do modelo proposto.

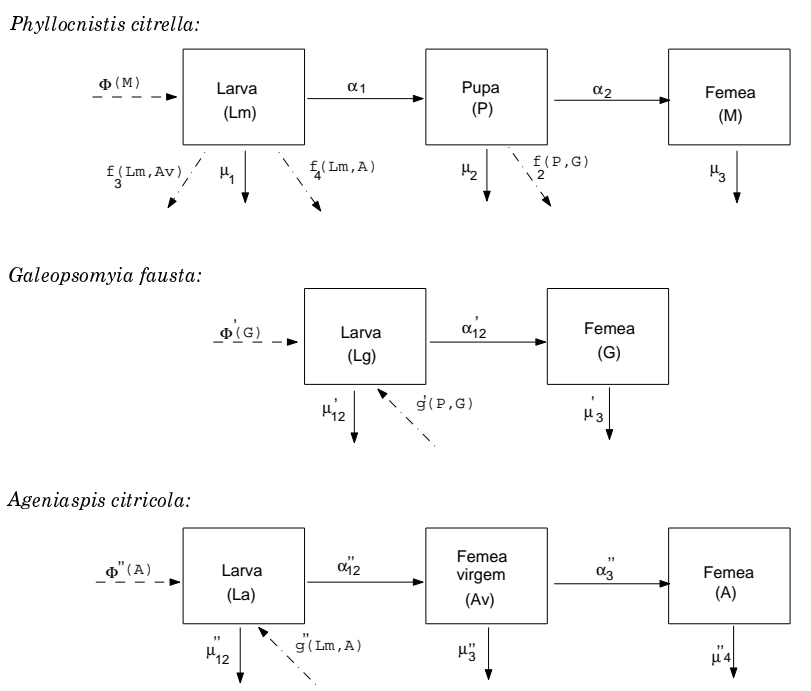

Figura 1: Esquema de compartimentos do modelo

Os parâmetros relacionados ao inseto-praga são $\alpha_{1}, \alpha_{2}, \mu_{1}, \mu_{2}$ e $\mu_{3}$, que são as taxas de, respectivamente, larvas que originam pupas, pupas que dão origem a adultos, mortalidade das fases de larva, pupa e indivíduo adulto. As funções $\Phi(M)$, $f_{2}(P, G), f_{3}\left(L_{m}, A_{v}\right)$ e $f_{4}\left(L_{m}, A\right)$ correspondem à taxa per capita de crescimento da população do inseto-praga e funções de parasitismo de fêmeas do parasito nativo e do parasito exótico (virgens e acasaladas) sobre o inseto-praga, respectivamente.

Os parâmetros relacionados ao parasito nativo são $\alpha_{12}^{\prime}, \mu_{12}^{\prime}$ e $\mu_{3}^{\prime}$, que são as taxas de, respectivamente, larvas do parasito nativo que, passando pela fase de pupa, vão originar indivíduos adultos, mortalidade das fases de larva e pupa, e mortalidade do indivíduo adulto. As funções $\Phi^{\prime}(G)$ e $g^{\prime}(P, G)$ são, respectivamente, a taxa per capita de crescimento da população do parasito nativo e função de crescimento da população do parasito nativo decorrente do parasitismo. Observe que a fase de pupa está embutida nos parâmetros $\alpha_{12}^{\prime}$ e $\mu_{12}^{\prime}$.

Os parâmetros relacionados ao parasito exótico são $\alpha_{12}^{\prime \prime}, \alpha_{3}^{\prime \prime}, \mu_{12}^{\prime \prime}, \mu_{3}^{\prime \prime}$ e $\mu_{4}^{\prime \prime}$, que são as taxas de, respectivamente, larvas do parasito que, passando pela fase de pupa, vão originar fêmeas virgens, fêmeas virgens que se acasalaram, mortalidade das fases de larva e pupa, mortalidade de fêmeas virgens e mortalidade de fêmeas acasaladas. As funções $\Phi^{\prime \prime}(A)$ e $g^{\prime \prime}\left(L_{m}, A\right)$ são, respectivamente, a taxa per capita de crescimento da população do parasito exótico e função de crescimento da população do parasito exótico decorrente do parasitismo. A fase de pupa está embutida nos parâmetros $\alpha_{12}^{\prime \prime}$ e $\mu_{12}^{\prime \prime}$.

As funções $f_{2}(\cdot), f_{3}(\cdot), f_{4}(\cdot), g^{\prime}(\cdot)$ e $g^{\prime \prime}(\cdot)$ representam a interação entre as espécies. Essa interação depende, por exemplo, do encontro entre as duas po- 
pulações (inseto-praga e parasito), da capacidade de busca do parasito e de sua especificidade. Assim, é assumido existir um encontro aleatório entre a fêmea da G. fausta e a pupa do inseto-praga, e entre as fêmeas virgens e acasaladas de $A$. citricola e a larva do inseto-praga. Devido a esses encontros, ocorre um decréscimo na população de pupas e larvas da $P$. citrella, proporcional às taxas $k_{2}, k_{3}$ e $k_{4}$, respectivamente. Em contraposição, as populações de pupas do parasito nativo e de larvas do parasito exótico são beneficiadas por esse encontro aleatório, de modo proporcional às taxas $k_{1}^{\prime}$ e $k_{1}^{\prime \prime}$, respectivamente. Essas aproximações são razoavelmente boas quando as populações são abundantes, o que pode não ocorrer na prática. Tais funções são dadas por

$$
\left\{\begin{array}{l}
f_{2}(P, G)=k_{2} P G, \\
f_{3}\left(L_{m}, A_{v}\right)=k_{3} L_{m} A_{v}, \\
f_{4}\left(L_{m}, A\right)=k_{4} L_{m} A, \\
g^{\prime}(P, G)=k_{1}^{\prime} P G \\
g^{\prime \prime}\left(L_{m}, A\right)=k_{1}^{\prime \prime} L_{m} A .
\end{array}\right.
$$

As taxas de crescimento natural $\Phi(\cdot), \Phi^{\prime}(\cdot)$ e $\Phi^{\prime \prime}(\cdot)$ caracterizam as diferentes considerações sobre a dinâmica vital. O modelo considera que as três populações crescem da mesma forma e são controladas pela capacidade de saturação do meio ambiente, ou seja, seguem o crescimento logístivo de Verhulst,

$$
\left\{\begin{array}{l}
\Phi(M)=\phi\left(1-\frac{M}{c}\right), \\
\Phi^{\prime}(G)=\phi^{\prime}\left(1-\frac{G}{c^{\prime}}\right), \\
\Phi^{\prime \prime}(A)=\phi^{\prime \prime}\left(1-\frac{A}{c^{\prime \prime}}\right),
\end{array}\right.
$$

onde $\phi, \phi^{\prime}$ e $\phi^{\prime \prime}$ correspondem à taxaa de, respectivamente, ovos da fêmea do insetopraga que originam larvas, de ovos da fêmea do parasito nativo que originam pupas e de ovos da fêmea acasalada do parasito exótico que originam larvas. Os parâmetros $c>0, c^{\prime}>0$ e $c^{\prime \prime}>0$ referem-se aos limites superiores que as populações $M, G$ e $A$, respectivamente, atingem decorrentes da capacidade de saturação do meioambiente.

Para o estudo analítico das três populações em interação, considera-se ainda que: (a) a hipótese de que o crescimento da população do parasito exótico só é possível na presença do inseto-praga, por ser um parasito específico, corresponde a se ter $\phi^{\prime \prime}=0$, ou seja, o crescimento intrínseco da população é nulo; (b) a capacidade de parasitismo das fêmeas virgens $\left(A_{v}\right)$ do parasito exótico é semelhante à capacidade das fêmeas acasaladas $(A)$. Tal hipótese corresponde a $k_{3}=k_{4} ;$ (c) o benefício do processo de parasitismo na população de larvas do parasito nativo é proporcional ao prejuízo na população de pupas do inseto-praga, ou seja, $k_{1}^{\prime}=a k_{2}$, com $0<a<1$ e (d) de modo semelhante, o benefício do processo de parasitismo na população de larvas do parasito exótico é proporcional ao prejuízo na população de larvas do inseto-praga, ou seja, $k_{1}^{\prime \prime}=a k_{4}$, com $0<a<1$.

Assim, considerando as hipóteses anteriores e as equações (2.1) e (2.2), o modelo matemático incorporando a dinâmica vital das três populações em interação, repre- 
sentado na Figura 1, é descrito por um sistema composto de 8 equações diferenciais ordinárias não-lineares dado por

$$
\left\{\begin{array}{l}
\frac{d L_{m}}{d t}=\phi\left(1-\frac{M}{c}\right) M-\left(\alpha_{1}+\mu_{1}\right) L_{m}-k_{4} L_{m}\left(A_{v}+A\right), \\
\frac{d P}{d t}=\alpha_{1} L_{m}-\left(\alpha_{2}+\mu_{2}\right) P-k_{2} P G \\
\frac{d M}{d t}=\alpha_{2} P-\mu_{3} M \\
\frac{d L_{g}}{d t}=\phi^{\prime}\left(1-\frac{G}{c^{\prime}}\right) G-\left(\alpha_{12}^{\prime}+\mu_{12}^{\prime}\right) L_{g}+a k_{2} P G \\
\frac{d G}{d t}=\alpha_{12}^{\prime} L_{g}-\mu_{3}^{\prime} G \\
\frac{d L_{a}}{d t}=-\left(\alpha_{12}^{\prime \prime}+\mu_{12}^{\prime \prime}\right) L_{a}+a k_{4} L_{m} A \\
\frac{d A_{v}}{d t}=\alpha_{12}^{\prime \prime} L_{a}-\left(\alpha_{3}^{\prime \prime}+\mu_{3}^{\prime \prime}\right) A_{v} \\
\frac{d A}{d t}=\alpha_{3}^{\prime \prime} A_{v}-\mu_{4}^{\prime \prime} A .
\end{array}\right.
$$

Tal sistema é explorado analiticamente na próxima seção.

\section{Análise do modelo}

O sistema de equações (2.3) representa a dinâmica das populações de fêmeas do inseto-praga, parasito nativo e parasito exótico em interação. Tal sistema é estudado em regime estacionário e seus pontos de equilíbrio, com respectivas condições de existência e estabilidade local, são determinados analiticamente.

A análise em regime estacionário do sistema de equações (2.3) para as populações em interação $\left(k_{2} \neq 0\right.$ e $\left.k_{4} \neq 0\right)$ apresenta 6 pontos de equilíbrio. O primeiro é a solução trivial, que representa a ausência ou extinção das populações, com condição de existência e estabilidade dada por $R<1$ e $R^{\prime}<1$, com

$$
R=\frac{\alpha_{1} \alpha_{2} \phi}{\mu_{3}\left(\alpha_{1}+\mu_{1}\right)\left(\alpha_{2}+\mu_{2}\right)} \quad \text { e } \quad R^{\prime}=\frac{\alpha_{12}^{\prime} \phi^{\prime}}{\mu_{3}^{\prime}\left(\alpha_{12}^{\prime}+\mu_{12}^{\prime}\right)},
$$

onde $R$ refere-se à população do inseto-praga e $R^{\prime}$ ao parasito nativo.

Os demais pontos referem-se ao domínio de uma população (e extinção das demais espécies) e a coexistência entre 2 ou 3 espécies. O estudo analítico de tais populações é apresentado a seguir.

\subsection{Domínio de uma única população}

O primeiro ponto de equilíbrio não trivial para as populações em interação refere-se à presença única do inseto-praga e extinção da população dos dois parasitos. Tal ponto é dado por

$$
N_{1}^{*} \equiv\left(\frac{c \mu_{3}\left(\alpha_{2}+\mu_{2}\right)}{\alpha_{1} \alpha_{2}}\left(1-\frac{1}{R}\right), \frac{c \mu_{3}}{\alpha_{2}}\left(1-\frac{1}{R}\right), c\left(1-\frac{1}{R}\right), 0,0,0,0,0\right),
$$


com condições de existência e estabilidade dadas por $R>1$ e $R^{\prime}<1$.

$\mathrm{O}$ segundo ponto de equilíbrio não trivial refere-se à presença única do parasito nativo e extinção das populações do inseto-praga e parasito exótico. Esse ponto é expresso por

$$
N_{2}^{*} \equiv\left(0,0,0, \frac{c^{\prime} \mu_{3}^{\prime}}{\alpha_{12}^{\prime}}\left(1-\frac{1}{R^{\prime}}\right), c^{\prime}\left(1-\frac{1}{R^{\prime}}\right), 0,0,0\right),
$$

com condições de existência e estabilidade dadas por $R<1$ e $R^{\prime}>1$.

Observa-se a inexistência do ponto de equilíbrio representando a presença única do parasito exótico, pois tal população só cresce na presença do inseto-praga, devido ao ganho no processo de parasitismo.

\subsection{Coexistência entre populações}

O sistema (2.3) possui o ponto de equilíbrio $N_{3}^{*}$ que representa a coexistência entre o inseto-praga e parasito nativo, o ponto $N_{4}^{*}$ que representa a interação entre o inseto-praga e parasito exótico e no máximo dois pontos de equilíbrio para as três populações vivendo conjuntamente.

\subsubsection{Solução $N_{3}^{*}$ : inseto-praga e parasito nativo}

A solução não trivial $N_{3}^{*} \equiv\left(\overline{L_{m 3}}, \overline{P_{3}}, \overline{M_{3}}, \overline{L_{g 3}}, \overline{G_{3}}, 0,0,0\right)$ é dada por

$$
\left\{\begin{array}{l}
\overline{L_{m 3}}=\frac{1}{\left(\alpha_{1}+\mu_{1}\right)}\left[\phi\left(1-\frac{\overline{M_{3}}}{c}\right) \overline{M_{3}}\right] \\
\overline{P_{3}}=\frac{\mu_{3}}{\alpha_{2}} \overline{M_{3}}, \\
\overline{M_{3}} \\
=\frac{\alpha_{2}}{a k_{2} \mu_{3}}\left(1+\frac{\alpha_{1} \alpha_{2}^{2} \phi \phi^{\prime}}{a c c^{\prime} \mu_{3}^{2} k_{2}^{2}\left(\alpha_{1}+\mu_{1}\right)}\right)^{-1}\left[\frac{\alpha_{1} \alpha_{2} \phi \phi^{\prime}}{c^{\prime} \mu_{3} k_{2}\left(\alpha_{1}+\mu_{1}\right)}+\frac{\mu_{3}^{\prime}\left(\alpha_{12}^{\prime}+\mu_{12}^{\prime}\right)}{\alpha_{12}^{\prime}}-\frac{\phi^{\prime}\left(\alpha_{2}+\mu_{2}\right)}{k_{2} c^{\prime}}-\phi^{\prime}\right] \\
\overline{L_{g 3}}=\frac{\alpha_{1} \alpha 2 \mu_{3}^{\prime} \phi}{\alpha_{12}^{\prime} \mu_{3} k_{2}\left(\alpha_{1}+\mu_{1}\right)}\left(1-\frac{\overline{M_{3}}}{c}\right)-\frac{\left(\alpha_{2}+\mu_{2}\right)}{k_{2}} \\
\overline{G_{3}}=\frac{\alpha_{1} \alpha 2 \phi}{\mu_{3} k_{2}\left(\alpha_{1}+\mu_{1}\right)}\left(1-\frac{\overline{M_{3}}}{c}\right)-\frac{\left(\alpha_{2}+\mu_{2}\right)}{k_{2}} .
\end{array}\right.
$$

Observa-se que deve-se ter $\overline{L_{m 3}}>0, \overline{P_{3}}>0, \overline{M_{3}}>0, \overline{L_{g 3}}>0$ e $\overline{G_{3}}>0$ em (3.4), implicando, pela manipulação algébrica do termo à direita na expressão de $\overline{M_{3}}$, nas seguintes condições:

(a) se $R<1$ e $R^{\prime}<1$, ambas as populações se extingüem devido à baixa capacidade de reprodução das espécies e $N_{3}^{*} \equiv(0,0,0,0,0,0,0,0)$;

(b) se $R<1$ e $R^{\prime}>1$, a população do inseto-praga se extingüe devido à sua baixa capacidade de reprodução e $N_{3}^{*} \equiv N_{2}^{*}$;

(c) se $R>1, R^{\prime}>1$ e $k_{2}>R^{\prime}(1-R)\left(\alpha_{2}+\mu_{2}\right)\left[c^{\prime}\left(1-R^{\prime}\right)\right]^{-1}$, a população do inseto-praga se extingüe devido ao alto grau de parasitismo e $N_{3}^{*} \equiv N_{2}^{*}$; 
(d) se $R>1, R^{\prime}>1$ e $k_{2}<R^{\prime}(1-R)\left(\alpha_{2}+\mu_{2}\right)\left[c^{\prime}\left(1-R^{\prime}\right)\right]^{-1}$, a existência do ponto de equilíbrio $N_{3}^{*}$ é satisfeita e as populações mantêm-se em coexistência;

(e) se $R>1$ e $R^{\prime}<1$, a condição de existência de $N_{3}^{*}$ é sempre satisfeita.

A estabilidade local da solução estacionária $N_{3}^{*}$ é avaliada pelo Método do Autovalor-Autovetor, através das raízes $\lambda$ do polinômio característico

$$
\Psi(\lambda)=\operatorname{det}\left(J\left[N_{3}^{*}\right]-\lambda I\right)=0,
$$

onde $J\left[N_{3}^{*}\right]$ é a matriz jacobiana do sistema de equações (2.3) calculada no ponto $N_{3}^{*}$ e $I$ é a matriz identidade de ordem 8. Os critérios de Routh-Hurwitz para equação característica de oitavo grau são numerosos. Em [4] conjectura-se que estes critérios podem ser resumidos pelo termo independente, ou seja, se este for positivo, então todos os autovalores terão parte real negativa. Em (3.5), para que o termo independente do polinômio em $\lambda$ seja estritamente positivo deve-se ter

$$
k_{4}<\frac{\mu_{4}^{\prime \prime}\left(\alpha_{12}^{\prime \prime}+\mu_{12}^{\prime \prime}\right)\left(\alpha_{3}^{\prime \prime}+\mu_{3}^{\prime \prime}\right)}{a \alpha_{12}^{\prime \prime} \alpha_{3}^{\prime \prime} \overline{L_{m 3}}}
$$

e, conseqüentemente, $N_{3}^{*}$ é localmente e assintoticamente estável. Entretanto, esse ponto de equilíbrio não é satisfatório para o controle biológico, pois na prática, percebe-se que o inseto-praga pode atingir altos picos populacionais em determinadas épocas do ano, mostrando que apenas a presença do parasito nativo não é suficiente para manter a população da $P$. citrella abaixo do nível de dano econômico [6].

\subsubsection{Solução $N_{4}^{*}$ : inseto-praga e parasito exótico}

A solução não trivial $N_{4}^{*} \equiv\left(\overline{L_{m 4}}, \overline{P_{4}}, \overline{M_{4}}, 0,0, \overline{L_{a 4}}, \overline{A_{v 4}}, \overline{A_{4}}\right)$ é dada por

$$
\left\{\begin{array}{l}
\overline{L_{m 4}}=\frac{\mu_{3}\left(\alpha_{2}+\mu_{2}\right)}{\alpha_{1} \alpha_{2}} \overline{M_{4}}, \\
\overline{P_{4}}=\frac{\mu_{3}}{\alpha_{2}} \overline{M_{4}}, \\
\overline{M_{4}}=\frac{\alpha_{1} \alpha_{2} \mu_{4}^{\prime \prime}\left(\alpha_{12}^{\prime \prime}+\mu_{12}^{\prime \prime}\right)\left(\alpha_{3}^{\prime \prime}+\mu_{3}^{\prime \prime}\right)}{a k_{4} \alpha_{12}^{\prime \prime} \alpha_{3}^{\prime \prime} \mu_{3}\left(\alpha_{2}+\mu_{2}\right)}, \\
\overline{L_{a 4}}=\frac{\mu_{4}^{\prime \prime}\left(\alpha_{3}^{\prime \prime}+\mu_{3}^{\prime \prime}\right)}{\alpha_{12}^{\prime \prime} \alpha_{3}^{\prime \prime}} \overline{A_{4}}, \\
\overline{A_{v 4}}=\frac{\mu_{4}^{\prime \prime} \overline{A_{4}}}{\alpha_{3}^{\prime \prime}}, \\
\overline{A_{4}}=\frac{\alpha_{1} \alpha_{2} \alpha_{3}^{\prime \prime}}{k_{4} \mu_{3}\left(\alpha_{2}+\mu_{2}\right)\left(\alpha_{3}^{\prime \prime}+\mu_{4}^{\prime \prime}\right)}\left[\phi-\frac{\phi \alpha_{1} \alpha_{2} \mu_{4}^{\prime \prime}\left(\alpha_{12}^{\prime \prime}+\mu_{12}^{\prime \prime}\right)\left(\alpha_{3}^{\prime \prime}+\mu_{3}^{\prime \prime}\right)}{a c k_{4} \alpha_{12}^{\prime \prime} \alpha_{3}^{\prime \prime} \mu_{3}\left(\alpha_{2}+\mu_{2}\right)}-\frac{\mu_{3}\left(\alpha_{1}+\mu_{1}\right)\left(\alpha_{2}+\mu_{2}\right)}{\alpha_{1} \alpha_{2}}\right] .
\end{array}\right.
$$

Para que $\overline{L_{m 4}}>0, \overline{P_{4}}>0, \overline{M_{4}}>0, \overline{L_{m 4}}>0, \overline{A_{v 4}}>0$ e $\overline{A_{4}}>0$ em (3.6), pela manipulação algébrica do termo à direita na expressão de $\overline{A_{4}}$, deve-se ter

$$
k_{4}>\frac{\phi \alpha_{1}^{2} \alpha_{2}^{2}\left(\alpha_{12}^{\prime \prime}+\mu_{12}^{\prime \prime}\right)\left(\alpha_{3}^{\prime \prime}+\mu_{3}^{\prime \prime}\right)}{a k_{4} \alpha_{2}^{\prime \prime} \alpha_{3}^{\prime \prime} \mu_{3}^{\prime \prime}\left(\alpha_{1}+\mu_{1}\right)\left(\alpha_{2}+\mu_{2}\right)^{2}(R-1)} .
$$


Assim, se $R>1$ e $k_{4}$ obedece a expressão acima, a condição de existência do ponto de equilíbrio $N_{4}^{*}$ é satisfeita. Devido à complexidade da análise da estabilidade local desse ponto, semelhante ao citado em (3.5), verificou-se numericamente que quando tais condições de existência são satisfeitas, o ponto $N_{4}^{*}$ é localmente e assintoticamente estável. Para o controle biológico esse ponto de equilíbrio não é satisfatório, pois o parasito nativo é deslocado pelo exótico. Então, caso tal espécie não consiga se estabelecer no decorrer do tempo devido a fatores ambientais, a inexistência de inimigos naturais faria com que a população do inseto-praga voltasse a crescer rapidamente, causando prejuízos econômicos.

\subsubsection{Soluções para a coexistência entre as três espécies}

As soluções não triviais para o sistema $(2.3)$ em regime estacionário, com $k_{2} \neq 0$ e $k_{4} \neq 0$, são obtidas em termos de raízes de um polinômio em $\overline{M_{5}}\left(\operatorname{Pol}\left(\overline{M_{5}}\right)\right)$ de terceiro grau, e expressões para $\overline{L_{m 5}}, \overline{P_{5}}, \overline{L_{g 5}}, \overline{G_{5}}, \overline{L_{a 5}}, \overline{A_{v 5}}$ e $\overline{A_{5}}$ em função de $\overline{M_{5}}$ dadas por

$$
\left\{\begin{array}{l}
\overline{L_{m 5}}=\frac{\phi \alpha_{3}^{\prime \prime}}{\gamma\left(\overline{A_{5}}\right)}\left(1-\frac{\overline{M_{5}}}{c}\right) \overline{M_{5}}, \\
\overline{P_{5}}=\frac{\mu_{3}}{\alpha_{2}} \overline{M_{5}}, \\
\overline{L_{g 5}}=\frac{\mu_{3}^{\prime}}{\alpha_{12}^{\prime}}\left[c^{\prime}\left(1-\frac{1}{R^{\prime}}\right)+\frac{a c^{\prime} k_{2} \mu_{3}}{\phi^{\prime} \alpha_{2}} \overline{M_{5}}\right], \\
\overline{G_{5}}=c^{\prime}\left(1-\frac{1}{R^{\prime}}\right)+\frac{a c^{\prime} k_{2} \mu_{3}}{\phi^{\prime} \alpha_{2}} \overline{M_{5}}, \\
\overline{L_{a 5}}=\frac{a \phi k_{4} \alpha_{3}^{\prime \prime} \overline{A_{5}}}{\left(\alpha_{12}^{\prime \prime}+\mu_{12}^{\prime \prime}\right) \gamma\left(\overline{A_{5}}\right)}\left(1-\frac{\overline{M_{5}}}{c}\right) \overline{M_{5}}, \\
\overline{A_{v 5}}=\frac{\mu_{4}^{\prime \prime}}{\alpha_{3}^{\prime \prime}} \overline{A_{5}}, \\
\overline{A_{5}}=d_{0}+d_{1}{\overline{M_{5}}}+d_{2}{\overline{M_{5}}}^{-1}, \\
\operatorname{Pol}\left(\overline{M_{5}}\right)=e_{3}{\overline{M_{5}}}^{3}+e_{2}{\overline{M_{5}}}^{2}+e_{1} \overline{M_{5}}+e_{0}=0,
\end{array}\right.
$$

onde

$$
\begin{array}{ll}
\gamma\left(\overline{A_{5}}\right)=\alpha_{3}^{\prime \prime}\left(\alpha_{1}+\mu_{1}\right)+k_{4}\left(\alpha_{3}^{\prime \prime}+\mu_{4}^{\prime \prime}\right) \overline{A_{5}}, & d_{2}=\frac{\phi \phi^{\prime} \alpha_{1} \alpha_{2}^{2} \alpha_{3}^{\prime \prime}}{a c^{\prime} k_{2}^{2} k_{4} \mu_{3}^{2}\left(\alpha_{3}^{\prime \prime}+\mu_{4}^{\prime \prime}\right)}, \\
d_{0}=-c d_{1}-\frac{1}{c} d_{2}-\frac{\alpha_{3}^{\prime \prime}\left(\alpha_{1}+\mu_{1}\right)}{k_{4}\left(\alpha_{3}^{\prime \prime}+\mu_{4}^{\prime \prime}\right)}, & e_{0}=\frac{\phi \phi^{\prime} \alpha_{1} \alpha_{2}^{2} \mu_{4}^{\prime \prime}\left(\alpha_{3}^{\prime \prime}+\mu_{3}^{\prime \prime}\right)}{a c^{\prime} k_{2}^{2} \mu_{3}^{2}}, \\
d_{1}=\frac{\phi \phi^{\prime} \alpha_{2} \alpha_{12}^{\prime \prime}\left(\alpha_{3}^{\prime \prime}\right)^{2}\left[\mu_{3}\left(\alpha_{2}+\mu_{2}\right)-c^{\prime} k_{2} \mu_{3}\left(1-\frac{1}{R^{\prime}}\right)\right]}{c c^{\prime} k_{2}^{2} \mu_{3}^{2} \mu_{4}^{\prime \prime}\left(\alpha_{3}^{\prime \prime}+\mu_{4}^{\prime \prime}\right)\left(\alpha_{3}^{\prime \prime}+\mu_{3}^{\prime \prime}\right)\left(\alpha_{12}^{\prime \prime}+\mu_{12}^{\prime \prime}\right)}, & e_{3}=\frac{a \phi k_{4} \alpha_{12}^{\prime \prime} \alpha_{3}^{\prime \prime}}{c\left(\alpha_{12}^{\prime \prime}+\mu_{12}^{\prime \prime}\right)}, \\
e_{1}=\frac{k_{4} \mu_{4}^{\prime \prime}\left(\alpha_{3}^{\prime \prime}+\mu_{3}^{\prime \prime}\right)\left(\alpha_{3}^{\prime \prime}+\mu_{4}^{\prime \prime}\right)}{c \alpha_{3}^{\prime \prime}} d_{2}+\frac{c k_{4} \mu_{4}^{\prime \prime}\left(\alpha_{3}^{\prime \prime}+\mu_{4}^{\prime \prime}\right)\left(\alpha_{3}^{\prime \prime}+\mu_{3}^{\prime \prime}\right)}{\alpha_{3}^{\prime \prime}} d_{1}, & \\
e_{2}=\frac{k_{4} \mu_{4}^{\prime \prime}\left(\alpha_{3}^{\prime \prime}+\mu_{4}^{\prime \prime}\right)\left(\alpha_{3}+\mu_{3}^{\prime \prime}\right)}{\alpha_{2} \alpha_{3}^{\prime \prime}} d_{1}-\frac{a \phi k_{4} \alpha_{12}^{\prime \prime} \alpha_{3}^{\prime \prime}}{\left(\alpha_{12}^{\prime 2}+\mu_{12}^{\prime \prime}\right)} &
\end{array}
$$


Observa-se que $e_{0}, e_{3}>0$ e $e_{1}, e_{2}<0$ e, portanto, o polinômio em $\overline{M_{5}}$ possui no máximo 2 raízes reais positivas (Regra de Sinal de Descartes). Através de um algoritmo numérico é possível obter as raízes reais positivas do polinômio em $\overline{M_{5}}$ e os valores correspondentes às demais variáveis pela equação (3.7). Cada solução corresponde a um ponto de equilíbrio do modelo proposto.

Mais uma vez, devido à complexidade do estudo da estabilidade local desse ponto como em (3.5), verificou-se numericamente que, conforme os valores utilizados para os parâmetros biológicos do modelo, é possível obter duas raízes reais positivas para o polinômio $\overline{M_{5}}$. Nestes casos, a menor raíz possui equilíbrio instável (breaking point) e a maior representa um ponto de equilíbrio localmente e assintoticamente estável.

A interação entre as três espécies é a solução mais adequada ao controle biológico, pois as populações podem coexistir com um risco mínimo de ocorrência de prejuízos econômicos.

\section{Conclusões}

As condições de existência e estabilidade para as soluções que representam o domínio de uma única população, $N_{1}^{*}$ e $N_{2}^{*}$, são dadas exclusivamente em função dos valores dos parâmetros $R$ e $R^{\prime}$. Tais parâmetros representam a capacidade de produção de descendentes viáveis para o inseto-praga e parasito nativo, respectivamente, em um meio limitante. Assim, a predominância de apenas uma das populações resulta quando o valor da capacidade de reprodução correspondente for maior que 1, e a do outro, menor que 1.

Para ser possível a coexistência entre as espécies, representadas pelas soluções $N_{3}^{*}, N_{4}^{*}$ e $N_{5}^{*}$, além de condições sobre $R$ e $R^{\prime}$, há condições sobre os parâmetros $k_{2}$ e $k_{4}$, que se referem ao grau de parasitismo dos inimigos naturais nativo e exótico, respectivamente. Em especial, deve-se ter sempre $R>1$.

Por hipótese, a espécie $A$. citricola só cresce na presença da $P$. citrella. Por isso, são necessários altos níveis de populações do inseto-praga para que o parasito exótico inicie o crescimento de sua população e passe a controlar (parasitar) o inseto-praga. Durante este período, a presença do parasito nativo é fundamental para o controle do inseto-praga. Entretanto, como o exótico ataca uma fase anterior do ciclo de vida da $P$. citrella, logo após iniciar seu crescimento, a população do parasito nativo cai para índice próximo à sua capacidade de saturação do meio $\left(c^{\prime}\right)$.

Mas, dependendo dos valores dos parâmetros biológicos do modelo, verificouse por simulação (variando-se os parâmetros biológicos em função da temperatura média mensal) que podem ser necessários valores da ordem de $k_{4}=10 k_{2}$ para se observar tal fenômeno. Assim, se as condições do agroecossistema em análise não forem favoráveis ao parasito exótico (umidade relativa superior a $60 \%$ e temperaturas médias acima de $25^{\circ} \mathrm{C}$ [1]), o exótico pode não se estabelecer e a população do inseto-praga pode atingir índices referentes ao nível de dano econômico.

Portanto, a competição entre as populações dos dois inimigos naturais se dá de forma indireta através de suas capacidades de parasitismo $\left(k_{2}\right.$ e $\left.k_{4}\right)$, pois cada 
espécie ataca uma fase diferente do ciclo de vida do inseto-praga. Tais valores, juntamente com as estimativas dos parâmetros biológicos do modelo, são fundamentais para a existência das três populações e respectivo controle do inseto-praga.

Abstract. In this work we develop a compartmental and deterministic mathematical model to analyze the population dynamics of the pest Phyllocnistis citrella in interaction with its two natural enemies Galeopsomyia fausta and Ageniaspis citricola. By the analytical study of the system equation, we evaluate the coexistence among the three species and the indirect competition between the natural enemies.

\section{Referências}

[1] M.C.M. das Chagas, "Phyllocnistis citrella Stainton, 1856 (Lepidoptera: Gracillariidae): Bioecologia e Relação com o Cancro Cítrico", Tese de Doutorado, ESALQ, USP, Piracicaba, SP, 1999.

[2] M.C.M das Chagas, J.R.P. Parra, P. Milano, P.T. Yamamoto, S. Gravena, P.E.B. Paiva e L.A.N. de Sá, Introduction of Ageniaspis citricola (Hymenoptera: Encyrtidae) in Brazil: rearing techniques and its release in the State of São Paulo, Brazil, em "Abstracts of XXII International Enthomophagus Insects Workshop", 1999.

[3] V.A. Costa, L.A.N. de Sá, J. LaSalle, E.A.B. De Nardo, F. Arellano e L.C. Fuini, Indigenous parasitoids (Hym., Chalcidoidea) of Phyllocnistis citrella Stainton (Lep., Gracillariidae) in Jaguariúna, São Paulo State, Brazil: preliminary results, J. Appl. Entom., 123 (1999), 237-240.

[4] M.B.F. Leite, R.C. Bassanezi e H.M. Yang, The basic reproduction ratio for a model of directly trasmitted infections considering the virus charge and the immunological response, J. Math. Appl. Med. Biol., 17 (2000), 15-31.

[5] W.W. Murdoch, Ecological theory and biological control, em "New directions in biological control: alternatives for supressing agricultural pests and diseases: proceedings of a UCLA Colloquium held at Frisco, Colorado", A.R. Liss, New York, 1990.

[6] S. Silveira Neto, O. Nakano, D. Barbin e N.A.V. Nova, "Manual de Ecologia dos Insetos", Ceres, São Paulo, 1976.

[7] S. Ternes, "Modelagem e Simulação da Dinâmica Populacional da LarvaMinadora-da-Folha-dos-Citros em Interação com seus Inimigos Naturais", Tese de Doutorado, FEEC, UNICAMP, Campinas, SP, 2001. 